\title{
ARTICLE
}

DOI: 10.1038/s41467-017-01910-9 OPEN

\section{Manufacture of highly loaded silica-supported cobalt Fischer-Tropsch catalysts from a metal organic framework}

Xiaohui Sun ${ }^{1}$, Alma I. Olivos Suarez ${ }^{1}$, Mark Meijerink² ${ }^{2}$ Tom van Deelen², Samy Ould-Chikh (i) ${ }^{3}$, Jovana Zečević Krijn P. de Jong ${ }^{2}$, Freek Kapteijn ${ }^{1}$ \& Jorge Gascon (1) 1,3

The development of synthetic protocols for the preparation of highly loaded metal nanoparticle-supported catalysts has received a great deal of attention over the last few decades. Independently controlling metal loading, nanoparticle size, distribution, and accessibility has proven challenging because of the clear interdependence between these crucial performance parameters. Here we present a stepwise methodology that, making use of a cobalt-containing metal organic framework as hard template (ZIF-67), allows addressing this long-standing challenge. Condensation of silica in the Co-metal organic framework pore space followed by pyrolysis and subsequent calcination of these composites renders highly loaded cobalt nanocomposites ( $50 \mathrm{wt} . \% \mathrm{Co})$, with cobalt oxide reducibility in the order of $80 \%$ and a good particle dispersion, that exhibit high activity, $\mathrm{C} 5+$ selectivity and stability in Fischer-Tropsch synthesis.

\footnotetext{
${ }^{1}$ Catalysis Engineering, Chemical Engineering Department, Delft University of Technology, Van der Maasweg 9, 2629 HZDelft, The Netherlands. ${ }^{2}$ Inorganic Chemistry and Catalysis, Debye Institute for Nanomaterials Science, Utrecht University, Universiteitsweg 99, 3584 CGUtrecht, The Netherlands. ${ }^{3}$ King Abdullah University of Science and Technology, KAUST Catalysis Center, Advanced Catalytic Materials, Thuwal 23955, Saudi Arabia. Correspondence and requests for materials should be addressed to J.G. (email: jorge.gascon@kaust.edu.sa)
} 
M etal (oxide) nanoparticles are instrumental in the development of new applications: from the production of fuels and chemicals through catalytic processes ${ }^{1}$ to nanoelectronics ${ }^{2}$ and energy conversion and storage ${ }^{3}$. Because most chemical and electronic phenomena occur at the surface, the intrinsic properties of nanoparticles depend strongly on their size, spatial distribution and even on their shape $e^{4,5}$. In general, small nanoparticles show high surface energies and are thermally unstable and prone to aggregate into larger clusters ${ }^{6}$. To tackle this issue, a general strategy consists of the use of supports with high surface area and well-developed porosity (e.g., $\mathrm{SiO}_{2}$ and $\mathrm{Al}_{2} \mathrm{O}_{3}$ ) that stabilize and prevent nanoparticle aggregation ${ }^{5,7,8}$.

Ion-adsorption $^{9,} 10$, impregnation and subsequent drying ${ }^{7}$, or deposition-precipitation ${ }^{11,} 12$ are among the most commonly used methods for the preparation of supported nanoparticles. Metal loading, nanoparticle size, and distribution are the three most important parameters that define performance of supported nanoparticles. Although it would be ideal to control independently each one of these parameters, in reality a strong interdependence exists. For example, for the methods described above, metal loading and particle size usually go hand in hand as a result of the fact that bigger nanoparticles and/or clusters are formed when high metal loadings are used. This interdependence is a clear drawback for the development of more efficient nanoparticle based composites for application in, i.e., heterogeneous catalysis. Structure sensitive reactions such as Fischer-Tropsch synthesis (FTS) are an outstanding example. For this specific process, when Co is used as the active metal, catalytic activity and selectivity to long-chain hydrocarbons are maximized when nanoparticles in the order of $8-30 \mathrm{~nm}$ are used ${ }^{13-15}$. Because of this reason, impregnation is the most widely used method for the preparation of industrial FTS catalysts ${ }^{16,17}$. However, using this method the maximum metal loading usually achieved is not higher than a 20 wt. $\%$, 18, 19 . As an alternative, deposition-precipitation methods have been developed to achieve higher metal loadings ${ }^{20,21}$. Yet, a large fraction of irreducible species (i.e., metal silicates and/or aluminates) is formed, resulting in non-optimal utilization of the active phase (that requires to be in the metallic form under reaction conditions $)^{21,22}$. In this respect, it is not surprising that the development of alternative methods for the preparation of these composites is gaining a tremendous attention in both the open and patent literature.

Among the different strategies suggested in literature, the use of metal organic-frameworks (MOFs) as precursors for the synthesis of nanomaterials such as metal (oxide) nanoparticles ${ }^{23}$ 26 , porous silica ${ }^{27,28}$, or nanoporous carbons ${ }^{29}$ offers unrivaled design possibilities, as we also demonstrate in this work. Herein we report a multi-step approach for the preparation of highly loaded Co on silica FTS catalysts that circumvents the interdependence between metal loading, active site dispersion, and accessibility. By using this approach, highly loaded cobalt nanocomposites $(\sim 50 \mathrm{wt} . \% \mathrm{Co})$ with cobalt oxide reducibility in the order of $80 \%$ and good particle dispersion were synthesized and tested in FTS. These catalysts exhibit high activity, C5 + selectivity, and excellent stability.

\section{Results}

Catalyst synthesis and characterization. Figure 1 illustrates the followed synthetic procedure. We used the zeolitic imidazolateframework ZIF-67, containing a 30 wt.\% Co $\left(\mathrm{Co}(\mathrm{MeIm})_{2}, \mathrm{MeIm}\right.$ $=2$-methylimidazolate) and tetramethyl orthosilicate (TMOS) as starting materials for the synthesis of cobalt catalysts. In this approach, a TMOS impregnated ZIF-67 was first subjected to a wet $\mathrm{N}_{2}$ flow under ambient conditions to facilitate TMOS hydrolysis inside the pores of the MOF. The obtained ZIF$67 @ \mathrm{SiO}_{2}$ sample was then pyrolyzed at different temperatures in the range of 773-973 K under $\mathrm{N}_{2}$ for $4 \mathrm{~h}$, followed by calcination in air at $673 \mathrm{~K}$ for $2 \mathrm{~h}$. The catalysts after pyrolysis and calcination are denoted as $\mathrm{Co@C}-\mathrm{SiO}_{2}-T$ and $\mathrm{Co@SiO}-T$, respectively, with
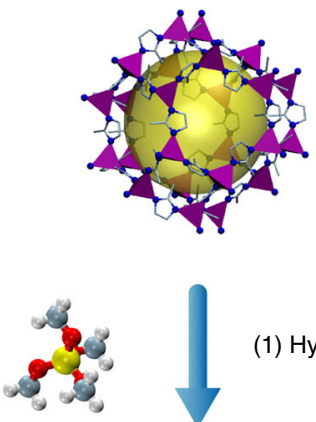

(1) Hydrolysis

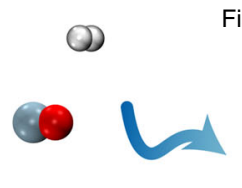

Fischer-Tropsch reaction
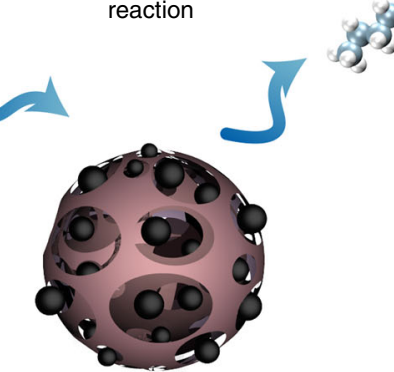
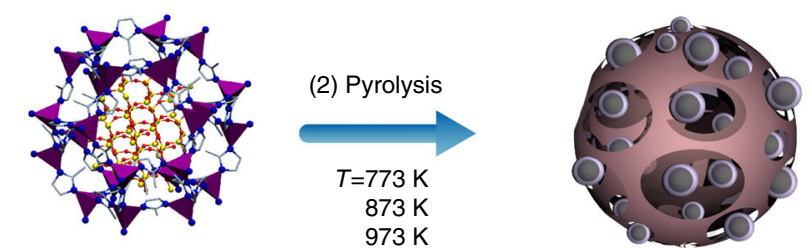

Fig. 1 Schematic illustration of the synthesis of the $\mathrm{Co@SiO} 2$ catalysts. (1) Impregnation and hydrolysis of TMOS molecules in the porosity of ZIF-67. (2)

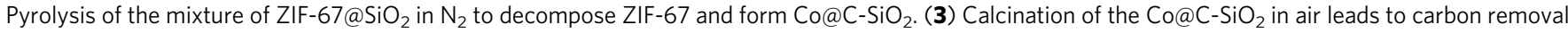

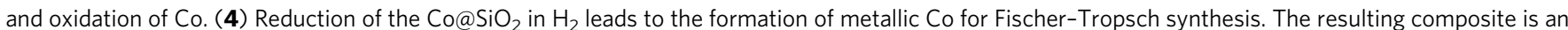
excellent catalyst for the low temperature Fischer-Tropsch synthesis 
a

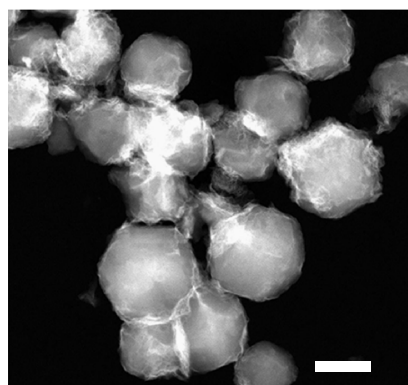

e

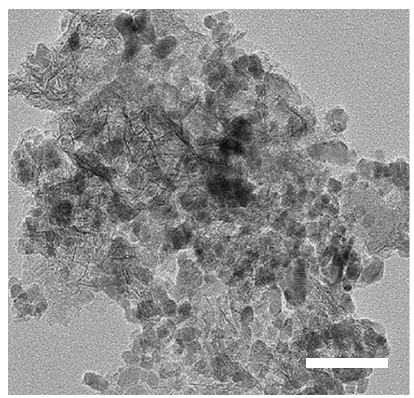

i

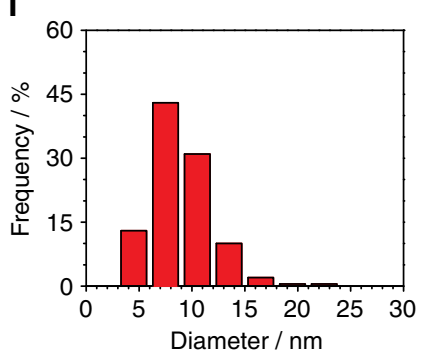

m

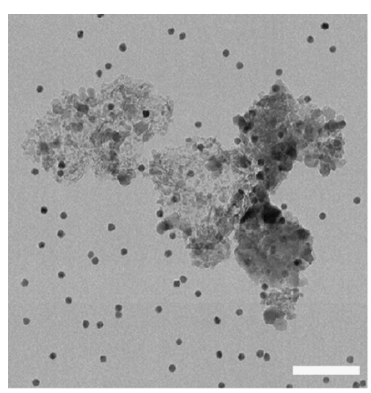

p

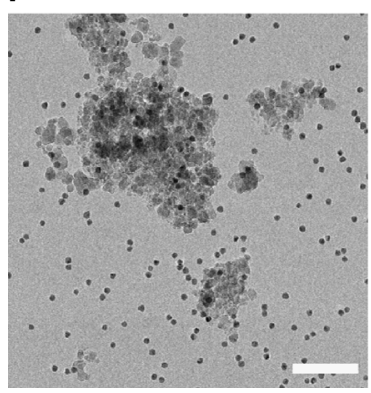

b

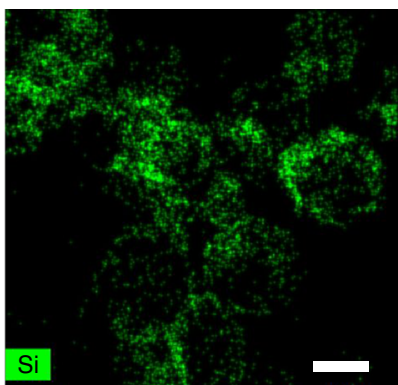

f
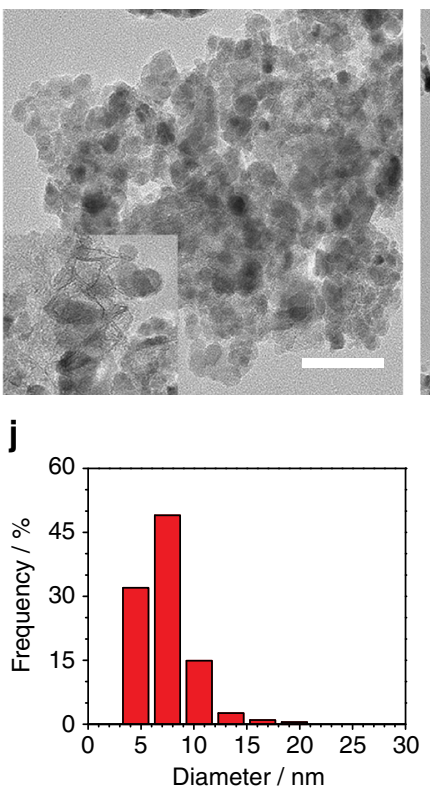

k
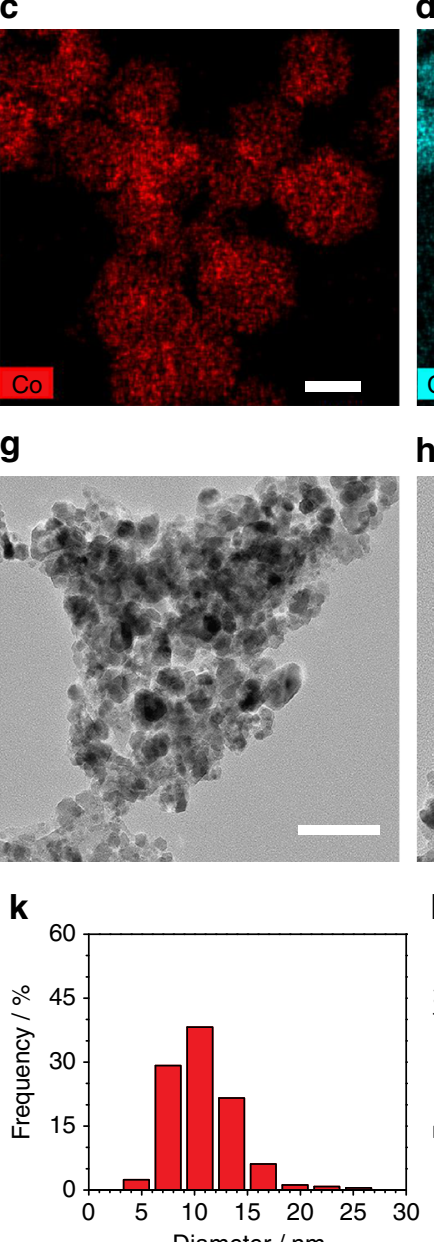

g

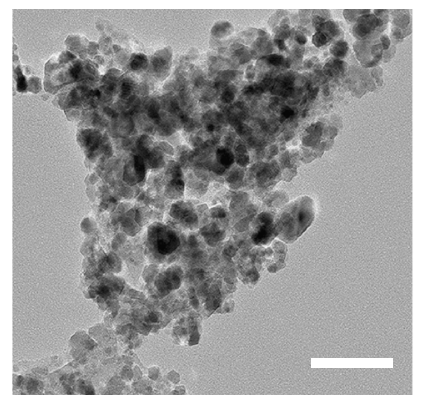

d

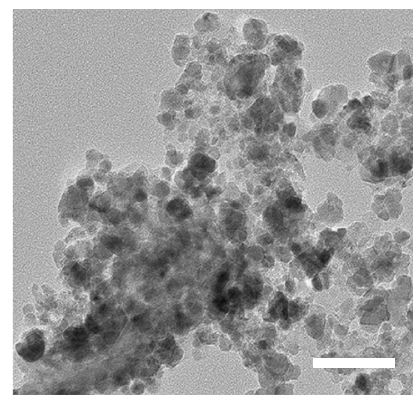

I

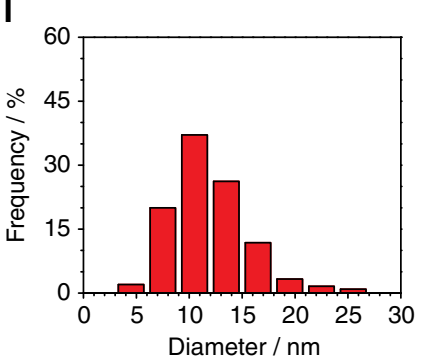

n

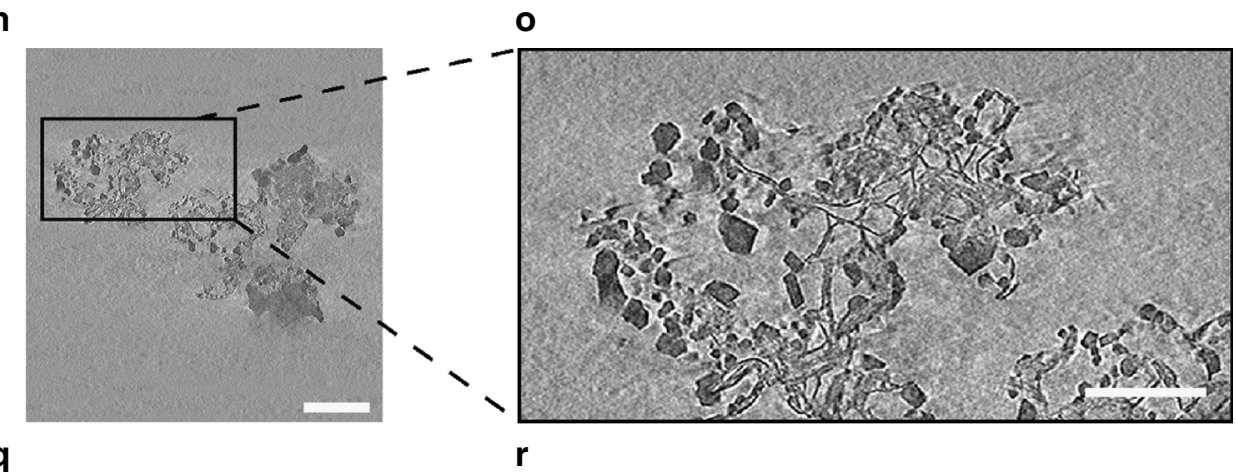

$\mathbf{q}$

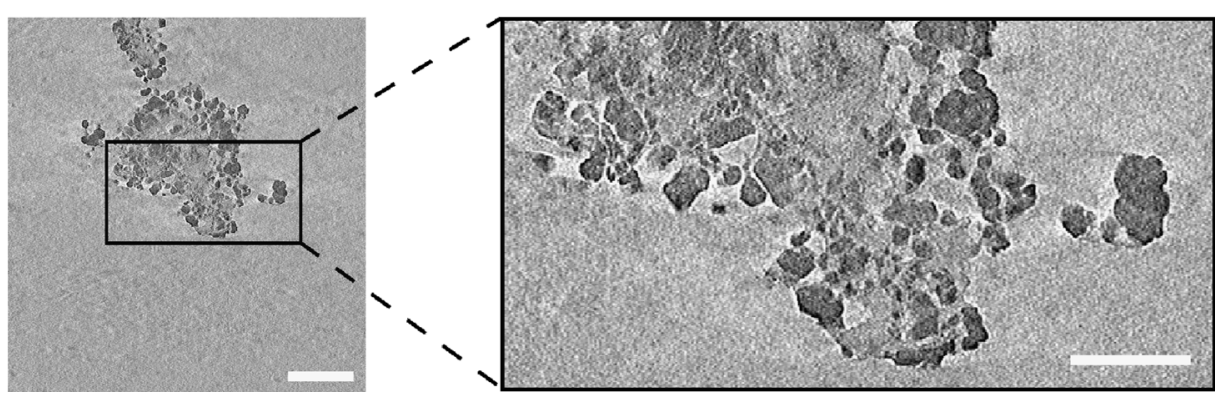

Fig. 2 Electron microscopy images and corresponding nanoparticle size distributions of cobalt based samples. a High-angle annular dark-field scanning electron (HAADF-STEM) micrograph of ZIF-67@SiO 2 (scale bar 200 nm). Elemental mapping of b Si, c Co, and d C in ZIF-67@SiO 2 sample (scale bars

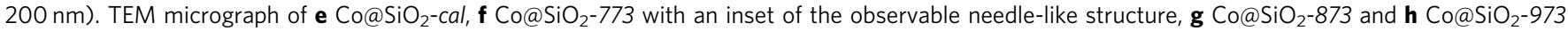
(scale bars from (e) - (h) 50 nm). Particle size histograms obtained from TEM analysis for i Co@SiO ${ }_{2}-c_{1}$, j Co@SiO ${ }_{2}-773, \mathbf{k}$ Co@SiO -873 , and I Co@SiO2973. Electron tomography results for m, n, o Co@SiO 2 -cal (scale bar 50,50, and 100 nm, respectively), and p, q, r Co@SiO $2-873$ (scale bar 50,50, and 100 $\mathrm{nm}$, respectively) 


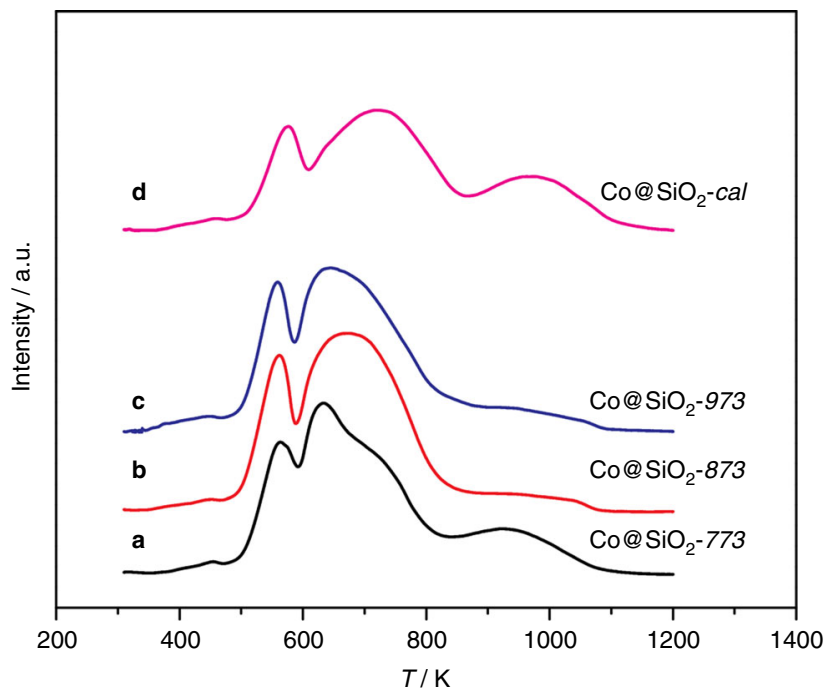

Fig. $3 \mathrm{TPR}\left(\mathrm{H}_{2}\right)$ profiles of $\mathrm{Co} @ \mathrm{SiO}_{2}$ catalysts. a Co@SiO -773 , b $\mathrm{Co} @ \mathrm{SiO}_{2}-$ 873, cCo@SiO -973 , and d Co@SiO2-cal.The TPR $\left(\mathrm{H}_{2}\right)$ experiments were performed from 303 to $1223 \mathrm{~K}$ at a ramp of $5 \mathrm{~K} \mathrm{~min}^{-1}$ in $10 \mathrm{vol} . \% \mathrm{H}_{2} / \mathrm{Ar}$

$T$ representing the pyrolysis temperature. For comparison, a

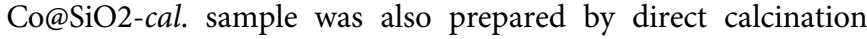
(skipping the intermediate pyrolysis step) of $\mathrm{ZIF}^{-67 @ S i \mathrm{O}_{2}}$ in air (details of the preparation process for all materials are shown in the Methods section). The Co loadings of the $\mathrm{Co@SiO}$ catalysts are $~ 50$ wt.\% (Supplementary Table 1).

The X-ray diffraction (XRD) pattern of the original ZIF-67 (Supplementary Fig. 1), confirms the structure of the MOF precursor ${ }^{30}$. Thermogravimetric (TG) analysis in $\mathrm{N}_{2}$ atmosphere of the hydrolyzed ZIF-67@SiO 2 indicates that the complete pyrolytic decomposition of the crystalline ZIF-67 occurs in the range of 800-850 K (Supplementary Fig. 2), further confirmed by XRD (Supplementary Fig. 3a). After the pyrolysis step, graphite $\left(2 \Phi=30.6^{\circ}\right)$ and metallic cobalt $\left(2 \Phi=51.8^{\circ}, 60.6^{\circ}\right)$ phases are formed. Notably, when a higher pyrolysis temperature is used, these peaks become much narrower and sharper, indicating a higher graphitization degree and a larger crystallite size of cobalt nanoparticles $^{31}$. After the additional calcination step, the characteristic peaks corresponding to ZIF-67, graphite, and metallic cobalt phases have disappeared and only the $\mathrm{Co}_{3} \mathrm{O}_{4}$ phase is observed (Supplementary Fig. 3b).

Both ZIF-67 and ZIF-67@SiO 2 display type-I $\mathrm{N}_{2}$ sorption isotherm (Supplementary Fig. 4a) typically associated with microporosity ${ }^{32}$. The Brunauer-Emmett-Teller area $\left(S_{\mathrm{BET}}\right)$ and pore volume $\left(V_{\mathrm{p}}\right)$ decreases from $1930 \mathrm{~m}^{2} \mathrm{~g}^{-1}$ and $0.71 \mathrm{~cm}^{3} \mathrm{~g}^{-1}$ to $1430 \mathrm{~m}^{2} \mathrm{~g}^{-1}$ and $0.56 \mathrm{~cm}^{3} \mathrm{~g}^{-1}$ after incorporation of $\mathrm{SiO}_{2}$ (Supplementary Table 1$)^{33}$. In contrast with the original ZIF$67 @ \mathrm{SiO}_{2}$, the $S_{\mathrm{BET}}$ and $V_{p}$ of all $\mathrm{Co@SiO} 2$ catalysts decreases drastically and exhibit type IV isotherms with type $\mathrm{H}_{3}$ hysteresis that closes at $P / P_{0} \approx 0.4$, suggesting the presence of a predominantly mesoporous structure which is the result of the agglomeration of small $\mathrm{SiO}_{2}$ particles (Supplementary Fig. 4b).

Transmission electron microscopy (TEM) and high-resolution transmission electron microscopy (HR-TEM) analysis in combination with elemental mapping (STEM/EDX (elemental energy dispersive X-ray)) give further information on the textural properties of the composites at different synthesis stages. Highangle annular dark-field scanning electron (HAADF-STEM) (Fig. 2a) analysis shows a well-defined rhombic dodecahedral morphology $(\sim 250 \mathrm{~nm})$ of the $\mathrm{ZIF}-67 @ \mathrm{SiO}_{2}$ catalysts similar to that of the original ZIF- $67^{30}$, whereas elemental mapping demonstrates an homogeneous dispersion of $\mathrm{Si}, \mathrm{Co}$ and $\mathrm{C}$

\section{Table 1 Average cobalt particle size and DOR of Co@SiO catalysts}

\begin{tabular}{|c|c|c|c|}
\hline Samples & $d_{\mathrm{CO}^{a}}{ }^{\mathrm{a}}$ & $d_{\mathrm{Co}^{b}}^{b}$ & DOR (\%) \\
\hline $\mathrm{Co@SiO}-773$ & 8.6 & 7.6 & 66 \\
\hline $\mathrm{Co@SiO}-873$ & 12.3 & 11.8 & 78 \\
\hline $\mathrm{Co@SiO}-973$ & 14.3 & 13.5 & 79 \\
\hline $\mathrm{Co} / \mathrm{SiO}_{2}$-cal. & 10.7 & 9.5 & 52 \\
\hline
\end{tabular}

${ }^{\mathrm{a}}$ Cobalt particle size is obtained from TEM analysis using at least $200 \mathrm{CO}_{3} \mathrm{O}_{4}$ nanoparticles and calculated from $\mathrm{Co}_{3} \mathrm{O}_{4}$ particle size using $\mathrm{Co}$ and $\mathrm{CO}_{3} \mathrm{O}_{4}$ densities

${ }^{b}$ Cobalt particle size is calculated from $\mathrm{H}_{2}$-chemisorption assuming the surface stoichiometry $\mathrm{H}$ /

$\mathrm{Co}=1$ and an atomic cross-sectional area of $0.0662 \mathrm{~nm}^{2}$. Cobalt oxide degree of reduction (DOR)

(Fig. 2b-d). After pyrolysis under $\mathrm{N}_{2}$ atmosphere, well dispersed cobalt nanoparticles in the carbon matrix can be observed in $\mathrm{Co@C-SiO}{ }_{2}-T$ samples (Supplementary Fig. 5a-c), with average particle size increasing from $5.4 \mathrm{~nm}$ in $\mathrm{Co@C}-\mathrm{SiO}_{2}-773$ to 11.0 $\mathrm{nm}$ in $\mathrm{Co@C}-\mathrm{SiO}_{2}-873$, and $13.3 \mathrm{~nm}$ in $\mathrm{Co@C}-\mathrm{SiO}_{2}-973$ (Supplementary Fig. 5d-f). According to HR-TEM, during pyrolysis, cobalt nanoparticles are encapsulated by multilayers of graphitic-carbon shells (Supplementary Fig. 5g-i) that render them, most likely, inaccessible. XRD analysis further confirm this observation, since reoxidation of most Co does not occur upon exposure to atmospheric conditions (vide supra). In addition, leaching experiment using $\mathrm{HCl}$ demonstrates that only a $30 \%$ of cobalt can be leached (Supplementary Table 2). The subsequent calcination removes the graphite shells and oxidizes metallic cobalt to $\mathrm{Co}_{3} \mathrm{O}_{4}$ (Fig. $3 \mathrm{f}-\mathrm{h}$, and Supplementary Fig. 6b-d), but hardly affects Co-particle size (Fig. 3j-1, and Table 1). No large cobalt clusters can be found in the $\mathrm{Co@SiO} 2-873$ sample even after reduction in $\mathrm{H}_{2}$ at $673 \mathrm{~K}$ for $10 \mathrm{~h}$ (Supplementary Fig. 7a-d). Interestingly, $\mathrm{Co@SiO} 2$-cal. (Fig. 2e, $\mathrm{m}$ and Supplementary Fig.8a) and $\mathrm{Co@SiO}{ }_{2}-773$ (the inset of Fig. 2f) show the presence of needle-like structures absent in samples pyrolyzed at higher temperatures. Additional analysis by combining TEM and EDX (Supplementary Fig. 8b, c) reveals the presence of both Si and Co in needle- rich areas and made us tentatively attribute this morphology to the formation of cobalt phyllosilicates ${ }^{34}$.

The reducibility of the metallic species in all calcined samples was studied by temperature-programmed reduction in $\mathrm{H}_{2}$ (TPR $\left.\left(\mathrm{H}_{2}\right)\right)$. All of the $\mathrm{Co@SiO} 2$ samples exhibit two overlapping reduction peaks centered at $\sim 570 \mathrm{~K}$ and $700 \mathrm{~K}$, and a broad reduction band between 850 and $1150 \mathrm{~K}$, as shown in Fig. 3. The first two peaks are ascribed to the two-step reduction of $\mathrm{Co}_{3} \mathrm{O}_{4}$ via $\mathrm{CoO}$ to metallic $\mathrm{Co}^{35}$, along with gasification of the residual carbon in the samples (Supplementary Fig. 9), whereas the broad feature illustrates the reduction of highly dispersed cobalt species in strong interaction with the $\mathrm{SiO}_{2}$ support (e.g., cobalt phyllosilicate $)^{36}$. In the case of $\mathrm{Co@SiO}$-cal., the second reduction occurs at a slightly higher temperature, indicative of a stronger interaction between cobalt nanoparticles and support, most likely due to the presence of very small cobalt particles, as proven from the electron tomography results in Fig. $2 \mathrm{~m}$. Moreover, the broad high-temperature band in Co@SiO2-cal. and $\mathrm{Co@SiO} \mathrm{S}_{2}-773$ implies the presence of a large fraction of irreducible cobalt silicates, in agreement with the TEM analysis above. This is further confirmed by the lower degree of reduction

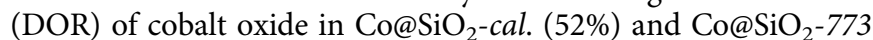
(66\%) than in $\mathrm{Co} @ S i O_{2}-873$ (78\%) and $\mathrm{Co@SiO} \mathrm{S}_{2}-973$ (79\%), see Table 1. These results highlight the importance of the intermediate pyrolysis step at a sufficiently high temperature as to achieve full destruction of the ZIF-67 sample to prevent the formation of irreducible cobalt silicate and therefore ensure an almost full utilization of the catalyst's cobalt loading. 
a

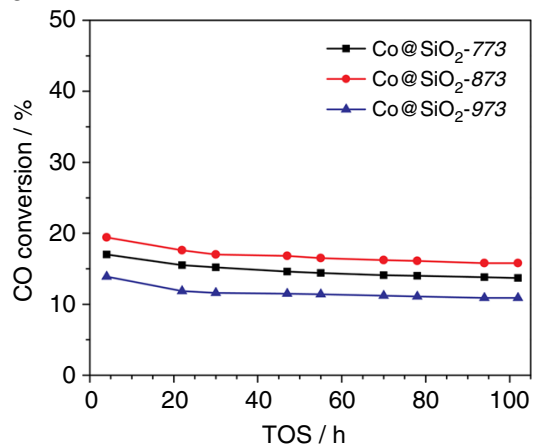

b

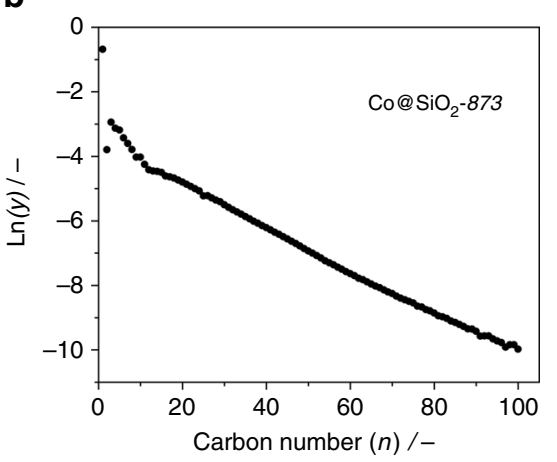

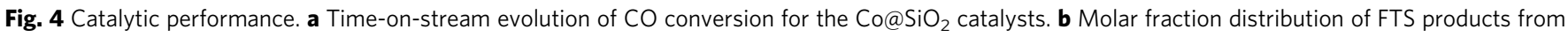
$\mathrm{Co@SiO}-873$ after $201 \mathrm{~h}$ on stream. Chain growth probability ( $\alpha=0.94$ ) obtained from the ASF plot in the C15-C100 hydrocarbon range. Reaction conditions: $483 \mathrm{~K}, 20$ bar, and $\mathrm{H}_{2} / \mathrm{CO}=1$, and syngas flow of $40 \mathrm{ml} \mathrm{min}^{-1}$

Table 2 Catalytic performance of $\mathrm{Co@SiO} 2$ catalysts after $102 \mathrm{~h}$ TOS

\begin{tabular}{|c|c|c|c|c|c|c|c|c|}
\hline \multirow[t]{2}{*}{ Sample } & \multirow[t]{2}{*}{ Sample weight (mg) } & \multirow{2}{*}{$\begin{array}{l}\text { Cobalt loading (wt. } \\
\%)\end{array}$} & \multirow[t]{2}{*}{$x_{\mathrm{co}}(\%)$} & \multirow[t]{2}{*}{ CTY $\left(10^{-5} \mathrm{~mol}_{\mathrm{Cog}} \mathrm{C}^{-1} \mathrm{Co}^{-1}\right)$} & \multirow{2}{*}{$\begin{array}{l}\text { TOF }\left(10^{-2} s\right. \\
-1)\end{array}$} & \multicolumn{3}{|c|}{$S(\%)$} \\
\hline & & & & & & C1 & C2-C4 & C5+ \\
\hline Co@SiOn-773 & 100 & 49 & 13.7 & 4.0 & 1.9 & 6.5 & 6.3 & 87.2 \\
\hline $\mathrm{Co@SiO} 2-873$ & 100 & 51 & 15.8 & 4.4 & 3.1 & 5.3 & 4.2 & 90.5 \\
\hline $\mathrm{Co@SiO}-973$ & 100 & 50 & 10.9 & 3.3 & 2.8 & 5.8 & 4.7 & 89.5 \\
\hline $\mathrm{Co} / \mathrm{SiO}_{2}-\mathrm{cal}$ & 100 & 46 & 10.6 & 3.3 & 1.9 & 7.5 & 6.8 & 85.7 \\
\hline
\end{tabular}

Catalytic results. The $\mathrm{Co} @ \mathrm{SiO}_{2}$ catalysts were tested in the FTS at $483 \mathrm{~K}, 20$ bar, $\mathrm{H}_{2} / \mathrm{CO}=1$, and a space velocity of $0.5 \mathrm{~mol}_{\mathrm{CO}} \mathrm{g}^{-1}$ cat. $\mathrm{h}^{-1}$. Figure $4 \mathrm{a}$ shows time-on-stream (TOS) evolution of CO conversion. All catalysts exhibit a good stability, and differences observed in activity are in line with the observed textural properties. $\mathrm{Co@SiO} \mathrm{S}_{2}-873$ displays the highest $\mathrm{CO}$ conversion, followed by $\mathrm{Co@SiO} 2-773$ and $\mathrm{Co@SiO}$-973. Table 2 summarizes cobalttime-yield (CTY), apparent turnover frequencies (TOF) and product selectivity for these catalysts after $102 \mathrm{~h}$ on stream. When CTY is plotted as a function of the pyrolysis temperature, a volcano-like curve is obtained, with an optimum for the sample pyrolyzed at $873 \mathrm{~K}$. The TOF values calculated for samples pyrolyzed at 873 and $973 \mathrm{~K}$ are similar and higher than that of the Co@SiO $2-773$ sample. The FTS process occurs on the surface of metallic cobalt nanoparticles with an optimal particle size around $10 \mathrm{~nm}$. On one hand, small cobalt nanoparticles normally possess a large fraction of low-coordinated surface sites (i.e., corner, kink, edge etc.), which to a large extent hamper CO dissociation and/or $\mathrm{CH}_{\mathrm{x}}$ hydrogenation ${ }^{13,37}$. Hence, we attribute the superior activity of $\mathrm{Co} @ \mathrm{SiO}_{2}-873$ to the high Co reducibility and the optimal Coparticle size (Table 1) ${ }^{13}, 14,16,38,39$. On the other hand, small cobalt nanoparticles have only few step sites, known for C-C formation towards long chain hydrocarbons, therefore resulting in a high methane selectivity ${ }^{40,41}$. Thus, the larger Co-particle size in the $\mathrm{Co} @ S i O_{2}-873$ and $\mathrm{Co}_{\mathrm{SiO}_{2}}-973$ samples when compared to $\mathrm{Co@SiO} 2-773$ results in a lower $\mathrm{CH}_{4}$ and a higher $\mathrm{C} 5+$ selectivity for these catalysts (Table 2), in excellent agreement with literature ${ }^{13}$. We argue that the low $\mathrm{H}_{2} / \mathrm{CO}$ ratio and operating temperature applied in this work $\left(\mathrm{H}_{2} / \mathrm{CO}=1,483 \mathrm{~K}\right)$ along with an optimal cobalt particle size in the synthesized $\mathrm{Co@SiO} 2^{-}$ 873 catalyst result in a chain growth probability $(\alpha)$ as high as $0.94^{42,43}$.

The performance of the $\mathrm{Co@SiO}$-cal. sample further emphasizes the key role of the intermediate pyrolysis step (Table 2). A high initial CO conversion over this sample along with a clear deactivation during the first $50 \mathrm{~h}$ on stream (Supplementary Fig. 10) is observed. We attribute the severe deactivation at the initial stage to the presence of a substantial amount of small cobalt nanoparticles $(<4 \mathrm{~nm})$, that are more susceptible to aggregation and/or oxidation than larger particles during highpressure FTS and which also more selective for the formation of $\mathrm{CH}_{4}{ }^{44}, 45$. In addition, although pyrolysis of Co-based MOFs under an inert atmosphere has recently been demonstrated as a promising route to prepare highly loaded Co@C hybrids with controllable cobalt particle size and distribution ${ }^{25}, 46-49$, these

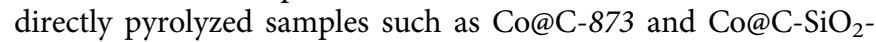
873 synthesized in this work show a poor activity and low C5 + selectivity along with an unacceptable $\mathrm{CH}_{4}$ selectivity in the FTS process under the same conditions as $\mathrm{Co@SiO} 2$ catalysts (Supplementary Fig. 11 and Supplementary Table 3). The inferior performance of these pyrolyzed samples can be ascribed to the inaccessibility of most cobalt nanoparticles, which are completely encapsulated by graphitic shells. (Supplementary Fig. 12 and Supplementary Table 2) ${ }^{50,51}$. Comparison of our results demonstrates the importance of the synthetic protocol here presented).

\section{Discussion}

The results here presented demonstrate that the stepwise hydrolysis-pyrolysis-calcination methodology is a promising route to synthesize highly loaded $\mathrm{Co@SiO}$ catalysts using ZIF-67 as a sacrificial template and TMOS as silicon source. During the high-temperature pyrolysis, the ZIF-67 structure decomposes, generating cobalt nanoparticles encapsulated by graphitic-carbon shells, which prevent the formation of large agglomerates, controlling in this way cobalt particle dispersity, whereas optimization of the pyrolysis temperature improves cobalt reducibility. 
a

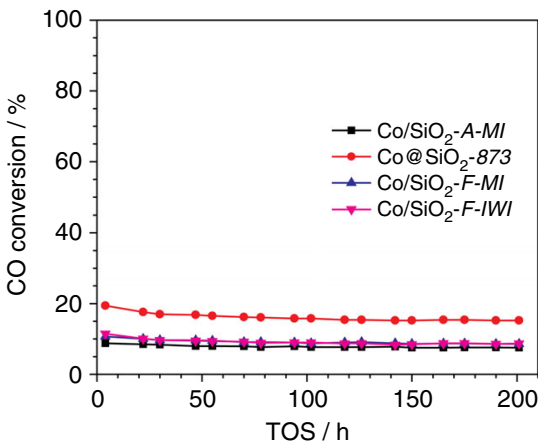

b

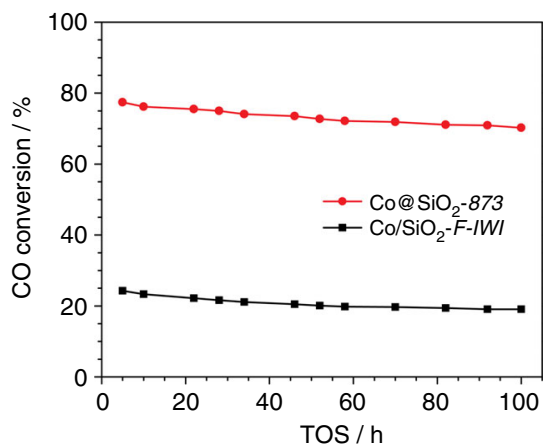

Fig. 5 Catalytic performance. a Time-on-stream evolution of $\mathrm{CO}$ conversion for the $\mathrm{Co} @ \mathrm{SiO}_{2}-873$ and $\mathrm{Co} / \mathrm{SiO}_{2}$ catalysts prepared using conventional methods. $M^{\prime}$ refers to melt infiltration. IWI refers to incipient wetness impregnation. A refers to Aerosil-200 support and $F$ refers to CARiACT Q-10 support. Reaction conditions: $483 \mathrm{~K}, 20$ bar, $\mathrm{H}_{2} / \mathrm{CO}=1$, and syngas flow of $40 \mathrm{ml} \mathrm{min}^{-1}$. b Time-on-stream evolution of $\mathrm{CO}$ conversion for the $\mathrm{Co@SiO} 2^{-}$ 873 and $\mathrm{Co} / \mathrm{SiO}_{2}-\mathrm{F}$ - TIWI catalysts prepared using two-step incipient wetness impregnation method (TIWI). Reaction conditions: $483 \mathrm{~K}, 26$ bar, $\mathrm{H}_{2} / \mathrm{CO}=2$, and syngas flow of $40 \mathrm{ml} \mathrm{min}^{-1}$

Table 3 Catalytic performance of $\mathrm{Co} @ \mathrm{SiO}_{2}-873$ and $\mathrm{Co} / \mathrm{SiO}_{2}$ catalysts prepared using conventional methods

\begin{tabular}{|c|c|c|c|c|c|c|c|c|}
\hline \multirow[t]{2}{*}{ Sample } & \multirow[t]{2}{*}{ Sample weight (mg) } & \multirow{2}{*}{$\begin{array}{l}\text { Cobalt loading (wt. } \\
\%)\end{array}$} & \multirow[t]{2}{*}{$x_{\mathrm{co}}(\%)$} & \multirow[t]{2}{*}{ 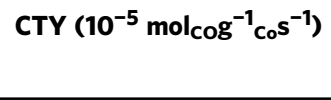 } & \multicolumn{4}{|c|}{$S(\%)$} \\
\hline & & & & & C1 & C2-C4 & C5+ & $\mathrm{CO} 2$ \\
\hline $\mathrm{Co@SiO}{ }_{2}-873^{a}$ & 100 & 51 & 15.2 & 4.2 & 5.2 & 3.8 & 91.0 & - \\
\hline $\mathrm{Co} / \mathrm{SiO}_{2}-\mathrm{F}-\mathrm{Ml}^{\mathrm{a}}$ & 100 & 42 & 8.6 & 3.0 & 4.8 & 4.3 & 90.9 & - \\
\hline $\mathrm{Co} / \mathrm{SiO}_{2}-\mathrm{F}-\left.\mathrm{IW}\right|^{\mathrm{a}}$ & 250 & 16.5 & 8.7 & 3.1 & 4.7 & 4.9 & 90.4 & - \\
\hline $\mathrm{Co@SiO}-873^{\mathrm{b}}$ & 175 & 51 & 70.2 & 7.8 & 9.7 & 5.2 & 84.7 & 0.4 \\
\hline $\mathrm{Co} / \mathrm{SiO}_{2}-\mathrm{F}-\mathrm{TIW} I^{\mathrm{b}}$ & & & 26.0 & 3.4 & 9.3 & 7.6 & $83.0^{c}$ & - \\
\hline
\end{tabular}

To further demonstrate the advantages of this synthetic methodology, we prepared additional highly loaded Co catalysts, with Co supported on commercially available Aerosil-200 (denoted as A) or CARiACT Q-10 (denoted as F) silica, by using melt infiltration (MI). Also two benchmark $\mathrm{Co} / \mathrm{SiO}_{2}$ catalysts with cobalt loading of 16 wt.\% and 32 wt.\%, respectively, were prepared by means of incipient wetness impregnation (IWI). The 32 wt. $\% \mathrm{Co} / \mathrm{SiO}_{2}-F-T I W I$ and 40 wt. $\% \mathrm{Co} / \mathrm{SiO}_{2}-A-M I$ catalysts consist mostly of large aggregates (Supplementary Fig. 13a-c, and Supplementary Fig. 14a, b) as a result of the lower versatility of the MI and IWI methods for high cobalt loadings. The comparison between the FTS performance of these catalysts and Co@SiO $\mathrm{S}_{2}-873$ is shown in Fig. 5a, b and Table 3. Under the studied conditions, the $\mathrm{Co@SiO}{ }_{2}-873$ displays a CTY at least 1.5 times $\left(\mathrm{H}_{2} / \mathrm{CO}=1\right)($ entry $1-4$, Table 3$)$ and/or 2.2 times $\left(\mathrm{H}_{2} / \mathrm{CO}\right.$ $=2$ ) (entry 5 and 7 , Table 3 ) higher than the other samples (in spite of the higher Co loading) and a comparable C5 + selectivity $(\sim 83 \%)$ to its $\mathrm{Co} / \mathrm{SiO}_{2}-\mathrm{F}-\mathrm{TIWI}$ counterpart at a similar $\mathrm{CO}$ conversion level $(\sim 26 \%)$. Interestingly, TEM images of the $\mathrm{Co@SiO} 2-873$ catalyst after $201 \mathrm{~h}$ TOS show a very good dispersion of cobalt nanoparticles on the $\mathrm{SiO}_{2}$ support along with very few aggregates (Supplementary Fig. 15a-d), in good agreement with the observed very mild catalyst deactivation with timeon stream. In comparison with other highly loaded catalysts prepared using traditional methods, the optimal particle size and high stability of cobalt nanoparticles in $\mathrm{Co@SiO} 2-873$ lead to more available cobalt sites and explain the high activity of $\mathrm{Co@SiO}-873$ in the FTS process ${ }^{7}$.
Overall, our results further highlight the potential and versatility of the use of MOFs as catalyst templates and opens the door to the controlled fabrication of highly loaded, accessible, active and stable metal supported catalysts thus coping with a major challenge in materials science and industrial catalysis.

\section{Methods}

Synthesis of the parent ZIF-67. In the synthesis of ZIF-67, $2.933 \mathrm{~g}$ of Co $\left(\mathrm{NO}_{3}\right)_{2} \cdot 6 \mathrm{H}_{2} \mathrm{O}$ and $6.489 \mathrm{~g}$ of 2-methylimidazole (MeIm) were separately dissolved in $200 \mathrm{ml}$ methanol. The latter clear solution was rapidly poured into the former pink solution with vigorous stirring for $24 \mathrm{~h}$ at room temperature. Afterwards, the bright purple products were collected by filtration, washed with methanol, and dried at $353 \mathrm{~K}$ for $10 \mathrm{~h}$ under vacuum.

Synthesis of ZIF-67@SiO ${ }_{\mathbf{2}}$. A total of $0.8 \mathrm{~g}$ of the synthesized ZIF-67 was immersed in $5 \mathrm{ml}$ TMOS in an autoclave, which was further transferred into a rotation oven and heated up to $333 \mathrm{~K}$ overnight. After the oven was cooled down to room temperature, the mixture was carefully washed with $1 \mathrm{ml}$ ethanol to remove the excess TMOS on the external surface of ZIF- 67 by filtration. Then the purple material was placed in a cotton thimble of $22 \mathrm{~mm}$ diameter and placed in a glass tube of $25 \mathrm{~mm}$ diameter. The glass tube was fitted to a round bottom flask containing $500 \mathrm{ml}$ of water. A needle to bubble the water with $10 \mathrm{ml} \mathrm{min}^{-1}$ of $\mathrm{N}_{2}$ flux was also fitted. The temperature was raised to $323 \mathrm{~K}$ to create a wet $\mathrm{N}_{2}$ stream to directly hydrolyze the TMOS molecules for $30 \mathrm{~h}$, followed by air drying at $333 \mathrm{~K}$ and vacuum drying at $373 \mathrm{~K}$ for $10 \mathrm{~h}$, successively. The obtained sample was denoted as ZIF-67@SiO 2 .

Synthesis of Co@C-SiO $2-$ T. A total of $0.8 \mathrm{~g}$ of ZIF-67@SiO ${ }_{2}$ were transferred into a quartz tubular reactor $(\sim L=1.0 \mathrm{~m} \times \mathrm{ID}=5.0 \mathrm{~cm})$ horizontally situated in a ceramic fiber oven (Carbolite, Sheffield). The reactor was flushed with $\mathrm{N}_{2}$ at $303 \mathrm{~K}$ for $0.5 \mathrm{~h}$, followed by direct carbonization at different temperature for $4 \mathrm{~h}$ under $\mathrm{N}_{2}$ 
$\left(150 \mathrm{ml} \mathrm{min}{ }^{-1}\right)$ at a ramp of $2 \mathrm{~K} \mathrm{~min}^{-1}$. The obtained sample was denoted as Co@C-SiO $2-T$, where $T(T=773,873,973 \mathrm{~K})$ refers to the pyrolysis temperature.

Synthesis of Co@SiO $-\mathbf{T}$ and $\mathbf{C o @ S i O}$-cal. The obtained Co@C-SiO ${ }_{2}-T$ samples were further calcined at $673 \mathrm{~K}$ in air $\left(150 \mathrm{ml} \mathrm{min}^{-1}\right)$ for $2 \mathrm{~h}$ at a ramp of $1 \mathrm{~K} \mathrm{~min}^{-1}$, and denoted as $\mathrm{Co@SiO} 2-T$, where $T(T=773,873,973 \mathrm{~K})$ refers to the pyrolysis temperature. For comparison, $0.8 \mathrm{~g}$ of ZIF-67@SiO ${ }_{2}$ was directly calcined at $673 \mathrm{~K}$ in air $\left(150 \mathrm{ml} \mathrm{min}^{-1}\right)$ for $2 \mathrm{~h}$ at a ramp of $1 \mathrm{~K} \mathrm{~min}^{-1}$, and this sample was denoted as $\mathrm{Co@SiO}{ }_{2}-c a l$.

Synthesis of Co@C-873. Co@C-873 was prepared by pyrolysis of $0.8 \mathrm{~g}$ ZIF-67 at

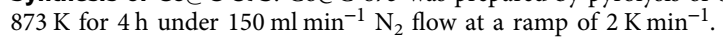

Synthesis of $\mathrm{Co} / \mathrm{SiO}_{2}$ catalysts with conventional methods. For the MI samples, $2.9 \mathrm{~g} \mathrm{Co}\left(\mathrm{NO}_{3}\right)_{2} \cdot 6 \mathrm{H}_{2} \mathrm{O}$ and $0.6 \mathrm{~g}$ of degassed $\mathrm{SiO}_{2}$ support (Aerosil-200 or CARiACT Q-10) were physically mixed in a mortar with a pestle under ambient conditions until the powder was homogeneously pink. Then the samples were transferred into a Teflon-lined steel autoclave and kept at $333 \mathrm{~K}$ for $24 \mathrm{~h}$, followed by calcination by heating to $673 \mathrm{~K}\left(1 \mathrm{~K} \mathrm{~min}^{-1}, 2 \mathrm{~h}\right)$ in a flow of air $\left(150 \mathrm{ml} \mathrm{min}^{-1}\right.$ for $0.8 \mathrm{~g}$ precursor loaded catalyst) in the same setup as mentioned above. The obtained samples were denoted as $\mathrm{Co} / \mathrm{SiO}_{2}-A-M I$ (Aerosil-200) and $\mathrm{Co} / \mathrm{SiO}_{2}-F-M$ (CARiACT Q-10), respectively. For the IWI sample, $1 \mathrm{~g}$ of degassed $\mathrm{SiO}_{2}$ suppor (CARiACT Q-10) was impregnated with $1 \mathrm{ml}$ of aqueous cobalt nitrate solution. The catalyst precursor was dried overnight under vacuum at $373 \mathrm{~K}$ followed by calcination by heating to $673 \mathrm{~K}\left(1 \mathrm{~K} \mathrm{~min}^{-1}, 2 \mathrm{~h}\right)$ in a flow of air $\left(150 \mathrm{ml} \mathrm{min}^{-1}\right.$ for $0.8 \mathrm{~g}$ precursor loaded catalyst) in the same setup as mentioned above. The obtained sample was denoted as $\mathrm{Co} / \mathrm{SiO}_{2}-F-I W I$. $\mathrm{Co} / \mathrm{SiO}_{2}-F-T I W I$ sample was prepared by two-step IWI of $\mathrm{Co}\left(\mathrm{NO}_{3}\right)_{2} \cdot 6 \mathrm{H}_{2} \mathrm{O}$ aqueous solution to $\mathrm{SiO}_{2}$ support (CARiACT Q-10), followed by drying overnight under vacuum at $373 \mathrm{~K}$, and calcination by heating to $673 \mathrm{~K}\left(1 \mathrm{~K} \mathrm{~min}^{-1}, 2 \mathrm{~h}\right)$ in a flow of air $\left(150 \mathrm{ml} \mathrm{min}^{-1}\right.$ for $0.8 \mathrm{~g}$ precursor loaded catalyst) in the same setup as mentioned above.

Characterization. The Co contents in the samples were measured by atomic adsorption spectroscopy (AAnalyst 200, Perkin Elmer, USA). PXRD patterns were measured by a Bruker D8 Advance X-ray diffractometer using monochromatic Co $K \alpha$ radiation $(\lambda=0.179026 \mathrm{~nm}) . \mathrm{N}_{2}$ adsorption-desorption isotherms were obtained using a Micromeritics Tristar 3020 at $77 \mathrm{~K}$, and samples were outgassed under vacuum at $423 \mathrm{~K}$ overnight prior to the analysis. For the analysis, the BET area was determined as outlined in Lange et al $^{33}$. The mesopore surface area was obtained from the $t$-plot applied to the $\mathrm{N}_{2}$ isotherm. TG analysis was carried out using a Mettler Toledo TGA/SDTA851e instrument by heating samples in $\mathrm{N}_{2}$ (100 $\mathrm{ml} \mathrm{min}^{-1}$ ) from room temperature to $1073 \mathrm{~K}$ at a ramp rate of $5 \mathrm{~K} \mathrm{~min}^{-1}$. TEM imaging and EDX mapping were performed on a JEM-2100 (JEOL) and a Talos F200X (FEI) microscopes operated at $200 \mathrm{kV}$. Tilt series of bright-field TEM images for electron tomography were taken with a Talos F200X (FEI) microscope over the angle range of $\pm 76^{\circ}$ with a tilt increment of $2^{\circ}$. Tilt series were aligned and reconstructed using IMOD software package ${ }^{52}$. Cobalt particle diameter $\left(d_{\mathrm{TEM}}\right)$ was calculated based on a minimum of 200 nanoparticles using the equation (1)

$$
d_{T E M}=\sum{ }_{i} n_{i} d_{i}^{3} / \sum_{i} n_{i} d_{i}^{2}
$$

where $n_{i}$ is the number of particles with diameter of $d_{i}$. The bright-field and HAADF-STEM imaging of the $\mathrm{Co} / \mathrm{SiO}_{2}-F-T I W I$ and spent $\mathrm{Co@SiO} \mathrm{O}_{2}-873$ catalysts were performed using a FEI TEM (model Titan 80-300 ST) at $300 \mathrm{kV}$. Temperature-programmed reduction in hydrogen $\left(\mathrm{TPR}\left(\mathrm{H}_{2}\right)\right)$ was performed in a flow of $10 \mathrm{vol} \% \mathrm{H}_{2} / \mathrm{Ar}\left(30 \mathrm{ml} \mathrm{min}^{-1}\right)$ at a heating rate of $5 \mathrm{~K} \mathrm{~min}^{-1}$ from ambient temperature to $1223 \mathrm{~K}$. The DOR was measured using TGA (Mettler Toledo TGA/ SDTA851e) in a flow of $10 \% \mathrm{H}_{2} / \mathrm{He}$. The samples were heated to $673 \mathrm{~K}$ and held there for $8 \mathrm{~h}$ (No weight loss was obtained after this time). After that the temperature was further increased to $1273 \mathrm{~K}\left(5 \mathrm{~K} \mathrm{~min}^{-1}\right)$. The DOR of cobalt was calculated using the equation (2)

$$
\left(n_{\text {Co }}^{\text {total }}-n_{C o}^{>673}\right) / n_{\text {Co }}^{\text {total }}
$$

A Micromeritics ASAP 2020 was used to measure $\mathrm{H}_{2}$-chemisorption. Samples dried at $100{ }^{\circ} \mathrm{C}$ were submitted to reduction in $\mathrm{H}_{2}$ at $673 \mathrm{~K}\left(10 \mathrm{~h}, 5 \mathrm{~K} \mathrm{~min}^{-1}\right)$ and evacuation at the same temperature. Isotherms were measured at $423 \mathrm{~K}$. The accesible cobalt surface areas were calculated assuming a one to one stoichiometry ( $\mathrm{H}: \mathrm{Co})$ and a Co-atomic cross section of $0.0662 \mathrm{~nm}^{2}$.

Catalytic testing. The FTS was carried out in a parallel 6-flow fixed-bed microreactor setup as previously described ${ }^{53}$. Certain amount of catalyst was mixed with $\mathrm{SiC}$ of similar size and loaded into a stainless steel tube lined with a quartz layer Catalysts were reduced in situ in pure $\mathrm{H}_{2}$ at $673 \mathrm{~K}$ for $10 \mathrm{~h}$ at $2 \mathrm{~K} \mathrm{~min}^{-1}$. Afterwards, the reactors were cooled to $453 \mathrm{~K}$ at which the pressure was increased to the target pressure ( 20 or 26 bar) under $\mathrm{H}_{2}$. Then, a $\mathrm{CO}$ flow was gradually introduced into the system, and finally reached an $\mathrm{H}_{2} / \mathrm{CO}$ ratio of 1 or 2 with syngas flow of $40 \mathrm{ml} \mathrm{min}^{-1}$. Next, the temperature was increased to the reaction temperature of
$483 \mathrm{~K}$ at $2 \mathrm{~K} \mathrm{~min}^{-1}$. The $\mathrm{C} 5+$ selectivity was calculated from the $\mathrm{CO}$ conversion by subtracting the fraction of $\mathrm{CO}$ used for the formation of $\mathrm{C}_{1}$ to $\mathrm{C}_{4}$ products, as determined via online GC (Hewlett Packard 5890, Series II) using $\mathrm{N}_{2}$ as an internal standard, from the total amount of CO converted.

Data availability. The authors declare that all other relevant data not included in the Supplementary Information and supporting the findings of this study are available on request.

Received: 9 March 2017 Accepted: 23 October 2017

Published online: 22 November 2017

\section{References}

1. Torres Galvis, H. M. et al. Supported iron nanoparticles as catalysts for sustainable production of lower olefins. Science 335, 835-838 (2012).

2. Poizot, P., Laruelle, S., Grugeon, S., Dupont, L. \& Tarascon, J. M. Nano-sized transition-metal oxides as negative-electrode materials for lithium-ion batteries. Nature 407, 496-499 (2000).

3. O'Regan, B. \& Gratzel, M. A low-cost, high-efficiency solar cell based on dyesensitized colloidal TiO2 films. Nature 353, 737-740 (1991).

4. Bell, A. T. The impact of nanoscience on heterogeneous catalysis. Science 299, 1688-1691 (2003)

5. Eggenhuisen, T. M., Breejen, J. Pd, Verdoes, D., Jongh, P. Ed \& Jong, K. Pd. Fundamentals of melt infiltration for the preparation of supported metal catalysts. The case of $\mathrm{Co} / \mathrm{SiO} 2$ for Fischer-Tropsch synthesis. J. Am. Chem. Soc. 132, 18318-18325 (2010).

6. Petit, C., Taleb, A. \& Pileni, M.-P. Self-Organization of magnetic nanosized cobalt particles. Adv. Mater. 10, 259-261 (1998).

7. Munnik, P., de Jongh, P. E. \& de Jong, K. P. Control and impact of the nanoscale distribution of supported cobalt particles used in Fischer-Tropsch catalysis. J. Am. Chem. Soc. 136, 7333-7340 (2014).

8. Espinosa-Alonso, L. et al. Tomographic energy dispersive diffraction imaging to study the genesis of $\mathrm{Ni}$ nanoparticles in 3D within $\gamma$ - $\mathrm{Al} 2 \mathrm{O} 3$ catalyst bodies. J. Am. Chem. Soc. 131, 16932-16938 (2009).

9. Schreier, M. \& Regalbuto, J. R. A fundamental study of Pt tetraammine impregnation of silica: 1 . The electrostatic nature of platinum adsorption. J. Catal. 225, 190-202 (2004).

10. Regalbuto, J. R., Navada, A., Shadid, S., Bricker, M. L. \& Chen, Q. An experimental verification of the physical nature of pt adsorption onto alumina. J. Catal. 184, 335-348 (1999).

11. Che, M., Cheng, Z. X. \& Louis, C. Nucleation and particle growth processes involved in the preparation of Silica-Supported nickel materials by a Two-Step procedure. J. Am. Chem. Soc. 117, 2008-2018 (1995).

12. Serp, P., Kalck, P. \& Feurer, R. Chemical vapor deposition methods for the controlled preparation of supported catalytic materials. Chem. Rev. 102, 3085-3128 (2002).

13. Bezemer, G. L. et al. Cobalt particle size effects in the fischer-tropsch reaction studied with carbon nanofiber supported catalysts. J. Am. Chem. Soc. 128, 3956-3964 (2006).

14. Prieto, G., Martínez, A., Concepción, P. \& Moreno-Tost, R. Cobalt particle size effects in Fischer-Tropsch synthesis: structural and in situ spectroscopic characterization on reverse micelle-synthesised Co/ITQ-2 model catalysts. J. Catal. 266, 129-144 (2009).

15. Barbier, A., Tuel, A., Arcon, I., Kodre, A. \& Martin, G. A. Characterization and catalytic behavior of $\mathrm{Co} / \mathrm{SiO} 2$ catalysts: influence of dispersion in the Fischer-Tropsch reaction. J.Catal. 200, 106-116 (2001).

16. Girardon, J.-S. et al. Effect of cobalt precursor and pretreatment conditions on the structure and catalytic performance of cobalt silica-supported Fischer-Tropsch catalysts. J. Catal. 230, 339-352 (2005).

17. Mile, B., Stirling, D., Zammitt, M. A., Lovell, A. \& Webb, M. The location of nickel oxide and nickel in silica-supported catalysts: Two forms of "NiO" and the assignment of temperature-programmed reduction profiles. J. Catal 114, 217-229 (1988).

18. Munnik, P., Velthoen, M. E. Z., de Jongh, P. E., de Jong, K. P. \& Gommes, C. J. Nanoparticle growth in supported nickel catalysts during methanation reaction -larger is better. Angew. Chem. 53, 9493-9497 (2014).

19. Munnik, P., Krans, N. A., de Jongh, P. E. \& de Jong, K. P. Effects of drying conditions on the synthesis of $\mathrm{Co} / \mathrm{SiO} 2$ and $\mathrm{Co} / \mathrm{Al} 2 \mathrm{O} 3$ Fischer-Tropsch catalysts. ACS Catal. 4, 3219-3226 (2014).

20. Nele, M., Vidal, A., Bhering, D. L., Carlos Pinto, J. \& Salim, V. M. M. Preparation of high loading silica supported nickel catalyst: simultaneous analysis of the precipitation and aging steps. Appl. Catal. A 178, 177-189 (1999). 
21. Bitter, J. H., van der Lee, M. K., Slotboom, A. G. T., van Dillen, A. J. \& de Jong K. P. Synthesis of highly loaded highly dispersed nickel on carbon nanofibers by homogeneous deposition-precipitation. Catal. Lett. 89, 139-142 (2003).

22. Bezemer, G. L. et al. Preparation of Fischer-Tropsch cobalt catalysts supported on carbon nanofibers and silica using homogeneous deposition-precipitation. $J$. Catal. 237, 291-302 (2006)

23. Hall, A. S., Kondo, A., Maeda, K. \& Mallouk, T. E. Microporous Brookite-Phase titania made by replication of a metal-organic framework. J. Am. Chem. Soc. 135, 16276-16279 (2013).

24. deKrafft, K. E., Wang, C. \& Lin, W. Metal-organic framework templated synthesis of $\mathrm{Fe} 2 \mathrm{O} 3 / \mathrm{TiO} 2$ nanocomposite for hydrogen production. Adv. Mater. 24, 2014-2018 (2012).

25. Santos, V. P. et al. Metal organic framework-mediated synthesis of highly active and stable Fischer-Tropsch catalysts. Nat. Commun. 6, 6451 (2015).

26. Čelič, T. B., Grilc, M., Likozar, B. \& Tušar, N. N. In situ generation of Ni nanoparticles from metal-organic framework precursors and their use for biomass hydrodeoxygenation. ChemSusChem 8, 1703-1710 (2015)

27. Kondo, A., Hall, A. S., Mallouk, T. E. \& Maeda, K. A new synthetic route to microporous silica with well-defined pores by replication of a metal-organic framework. Chemistry 21, 12148-12152 (2015)

28. Rieter, W. J., Taylor, K. M. L. \& Lin, W. Surface modification and functionalization of nanoscale metal-organic frameworks for controlled release and luminescence sensing. J. Am. Chem. Soc. 129, 9852-9853 (2007).

29. Liu, B., Shioyama, H., Akita, T. \& Xu, Q. Metal-Organic framework as a template for porous carbon synthesis. J. Am. Chem. Soc. 130, 5390-5391 (2008)

30. Banerjee, R. et al. High-throughput synthesis of zeolitic imidazolate frameworks and application to CO2 capture. Science 319, 939-943 (2008).

31. Torad, N. L. et al. Direct synthesis of MOF-Derived nanoporous carbon with magnetic Co nanoparticles toward efficient water treatment. Small 10, 2096-2107 (2014).

32. Tang, J. et al. Thermal conversion of core-shell metal-organic frameworks: a new method for selectively functionalized nanoporous hybrid carbon. J. Am. Chem. Soc. 137, 1572-1580 (2015).

33. De Lange, M. F., Vlugt, T. J. H., Gascon, J. \& Kapteijn, F. Adsorptive characterisation of porous solids: error analysis guides the way. Microporous Mesoporous Mater. 200, 199-215 (2014).

34. Kiss, G., Kliewer, C. E., DeMartin, G. J., Culross, C. C. \& Baumgartner, J. E. Hydrothermal deactivation of silica-supported cobalt catalysts in Fischer-Tropsch synthesis. J. Catal. 217, 127-140 (2003).

35. Sartipi, S. et al. Hierarchical H-ZSM-5-supported cobalt for the direct synthesis of gasoline-range hydrocarbons from syngas: Advantages, limitations, and mechanistic insight. J. Catal. 305, 179-190 (2013).

36. Puskas, I., Fleisch, T. H., Hall, J. B., Meyers, B. L. \& Roginski, R. T. Metalsupport interactions in precipitated, magnesium-promoted cobaltsilica catalysts. J. Catal. 134, 615-628 (1992).

37. den Breejen, J. P. et al. On the origin of the cobalt particle size effects in Fischer -Tropsch catalysis. J. Am. Chem. Soc. 131, 7197-7203 (2009).

38. Tsubaki, N., Sun, S. \& Fujimoto, K. Different functions of the noble metals added to cobalt catalysts for Fischer-Tropsch synthesis. J. Catal. 199, 236-246 (2001).

39. Khodakov, A. Y., Griboval-Constant, A., Bechara, R. \& Zholobenko, V. L. Pore size effects in fischer tropsch synthesis over Cobalt-Supported mesoporous silicas. J. Catal. 206, 230-241 (2002).

40. Van Santen, R. A. Complementary structure sensitive and insensitive catalytic relationships. Acc. Chem. Res. 42, 57-66 (2009).

41. Ralston, W. T., Melaet, G., Saephan, T. \& Somorjai, G. A. Evidence of structure sensitivity in the Fischer-Tropsch reaction on model cobalt nanoparticles by time-resolved chemical transient kinetics. Angew. Chem. 56, 7415-7419 (2017).

42. Espinoza, R. L., Steynberg, A. P., Jager, B. \& Vosloo, A. C. Low temperature Fischer-Tropsch synthesis from a sasol perspective. Appl. Catal. A 186, 13-26 (1999).

43. Vervloet, D., Kapteijn, F., Nijenhuis, J. \& van Ommen, J. R. Fischer-Tropsch reaction-diffusion in a cobalt catalyst particle: aspects of activity and selectivity for a variable chain growth probability. Catal. Sci. Technol. 2, 1221-1233 (2012).

44. van Steen, E. et al. Stability of nanocrystals: thermodynamic analysis of oxidation and re-reduction of cobalt in Water/Hydrogen mixtures. J. Phys. Chem. B. 109, 3575-3577 (2005).
45. Tsakoumis, N. E. et al. Evaluation of reoxidation thresholds for $\gamma-\mathrm{Al} 2 \mathrm{O} 3$ Supported cobalt catalysts under Fischer-Tropsch synthesis conditions. J. Am. Chem. Soc. 139, 3706-3715 (2017).

46. Wang, X. et al. MOF derived catalysts for electrochemical oxygen reduction. $J$ Mater.Chem. A 2, 14064-14070 (2014).

47. Zhou, Y.-X., Chen, Y.-Z., Cao, L., Lu, J. \& Jiang, H.-L. Conversion of a metalorganic framework to $\mathrm{N}$-doped porous carbon incorporating $\mathrm{Co}$ and $\mathrm{CoO}$ nanoparticles: direct oxidation of alcohols to esters. Chem. Commun. 51, 8292-8295 (2015)

48. Qiu, B. et al. Highly dispersed Co-based Fischer-Tropsch synthesis catalyst from metal-organic frameworks. J. Mater. Chem. A 5, 8081-8086 (2017).

49. Pei, Y., Li, Z. \& Li, Y. Highly active and selective Co-based Fischer-Tropsch catalysts derived from metal-organic frameworks. AIChE J. 63, 2935-2944 (2017)

50. Zhang, L. et al Co-N-C catalyst for $\mathrm{C}-\mathrm{C}$ coupling reactions: on the catalytic performance and active sites. ACS Catal. 6563-6572 (2015).

51. Sun, X. et al. Metal-Organic framework mediated Cobalt/Nitrogen-Doped carbon hybrids as efficient and chemoselective catalysts for the hydrogenation of nitroarenes. Chem. Cat. Chem 9, 1854-1862 (2017).

52. Kremer, J. R., Mastronarde, D. N. \& McIntosh, J. R. Computer visualization of three-dimensional image data using IMOD. J. Struct. Biol. 116, 71-76 (1996).

53. Sartipi, S. et al. Six-flow operations for catalyst development in Fischer-Tropsch synthesis: bridging the gap between high-throughput experimentation and extensive product evaluation. Rev. Sci. Instrum. 84, 124101 (2013).

\section{Acknowledgements}

We thank C. Hernandez-Mejia for additional FTS testing, H. Oosterbeek for the GC analysis and A. Dikhtiarenko for help in the design of the graphical abstract. X.S. acknowledges financial support from China Scholarship Council (CSC).

\section{Author contributions}

X.S. and J.G. conceived, coordinated the research and designed the experiments. X.S. and A.I.O.S. synthesized and characterized the catalysts. X.S. performed most of the catalytic testing and data interpretation. T.v.D. performed and analyzed $\mathrm{H}_{2}$ chemisorption. S.O. C., M.M. and J.Z. carried out and analyzed the microscopy characterization. The manuscript was primarily written by X.S. and J.G. with input from all authors. All authors participated in the discussion of the results.

\section{Additional information}

Supplementary Information accompanies this paper at doi:10.1038/s41467-017-01910-9.

Competing interests: The authors declare no competing financial interests.

Reprints and permission information is available online at http://npg.nature.com/ reprintsandpermissions/

Publisher's note: Springer Nature remains neutral with regard to jurisdictional claims in published maps and institutional affiliations.

(i) Open Access This article is licensed under a Creative Commons Attribution 4.0 International License, which permits use, sharing, adaptation, distribution and reproduction in any medium or format, as long as you give appropriate credit to the original author(s) and the source, provide a link to the Creative Commons license, and indicate if changes were made. The images or other third party material in this article are included in the article's Creative Commons license, unles indicated otherwise in a credit line to the material. If material is not included in the article's Creative Commons license and your intended use is not permitted by statutory regulation or exceeds the permitted use, you will need to obtain permission directly from the copyright holder. To view a copy of this license, visit http://creativecommons.org/ licenses/by/4.0/.

(C) The Author(s) 2017 